\title{
A Design of a Multi-Agent Smart E-Examiner
}

\author{
Khaled Nasser ElSayed \\ Computer Science Department, Umm AlQura University
}

\begin{abstract}
- this paper proposes a design of an application of multi agent technology on a semantic net knowledge base, to build a smart e-examiner system. This e-examiner could be used in building and grading a personalized special on-line eassessment. The produced e-assessment should cover the majority of examined topics and material. It should cover various levels of difficulties and learners profile(s). The e-examiner will use a semantic net question bank, to emphasize on the structuring categories of all course domains. This task is done through four different intelligent agents: control agent, personal agent, examiner agent, and grading agent. The system might select questions from a bank of questions for several courses. It could be used in different education levels and natures. Also, it will produce a key for the produced exam, to be used latter in grading, and giving final marks of e-assessments.
\end{abstract}

Keywords-m-Learning; e-Assessments; Multi-agent; Semantic net; Examiner

\section{INTRODUCTION}

The e-learning and m-learning are considered the important ways to respond to the needs of the d-learning. Many traditional learning styles should be applied together with the learner-centered approach to represent the needs of the e-learning system [1].

Software educational systems have become highly complex in both concept and design in the education of most sciences. In these systems, there is a wide difference between the role of the computer from solely a transmitter of knowledge and using it as a tool that aids in the construction of knowledge, as in [2].

There are an increasing number of $\mathrm{AI}$ algorithms and applications that are applied in tutoring and education systems. One of the AI techniques is the intelligent agent, which could be widely used in education. An intelligent agent is an autonomous calculated entity.

An intelligent agent could collect personal interests of the learner (s) and user (s) to give instance response according to the pre-specified demands of them. A personal one can discover their personal interests easily without disturbing them. So, it is suitable for personalized e-learning by recommending selected suitable learning materials [3].

An agent could detect by its sensors and perform actions on its environment by actuators to achieve target goals. The intelligent agent could learn new knowledge and uses it in achieving target goals. It might be simple procedure or program to perform simple goal or task. Also, it could be a complicated system working as a group of persons cooperated together towards a target goal [4].

A multi-agent system is constructed from a several intelligent agents, cooperated and interacted together to achieve target goal(s) within an environment. It could solve problems that are difficult be solved with an individual agent [5].

Adaptive learning is a learner-centric and individualized learning. The target of this learning may be certain learner or group of learner have a public feature(s). According to that, each learner could select chapters, exercises, and remedial actions even the curriculum relative to his individual's mental state. Then the learning system can maintain a dynamic knowledge for learners. It monitors the learner's behavior and stores his mental model which could be used in identifying the causes of the student performance [6].

Nowadays, there are many tools help in producing assessments. Advanced eLearning Builder [7] is an Authoring tool designed for creating e-learning materials such as e-tests, tutorials and quizzes in visual mode and compiling them in EXE form. Also, MicroGrade and Micro Test [8] are desktop applications with built-in access to hosted Internet sites for secure test delivery and grade posting for Windows and Macintosh compatible. While, OnlineTesting offers in [9] software and programming services to offer online quizzes and tests through the Internet or within an Intranet.

There are many other software tools for producing assessments like; ProPrfos [10], Adit Sofware [11], Quizworks [12] and ExamJet [13]. Majority of those software tools enable the human examiner to select questions or the question are selected randomly.

This paper presented a design of a multi-agent based smart e-examiner system. This design is for building a personalized e-assessment according to the study, level and profile of the learner(s). It builds its semantic net as object oriented knowledge base (question KB) to be filled with questions that cover the materials of topics of courses. Then it is running its four agents (control agent, personal agent, examiner agent and grading agent) on that question $\mathrm{KB}$ to select questions and building e-assessments according to learner(s) education level and profile data. The e-examiner agent gets its intelligence from watching and learning from the human examiner to enhance the selection of questions. It is designed for education in academic institutions, training centers, human resources departments. Also, it can help human examiners in producing their own assessments.

\section{CATEGORIES OF GENERATED QUESTIONS}

Questions are the infrastructure of the system, which could be generated for building an e-assessment. These questions and their answers from several e-courses construct the semantic net question KB. There are four categories of 
exercises; each includes a number of questions of that category.

- The Multiple Choice Category; where a question could be a text (sentence), while the answer is one correct choice from available four multiple choices (might be less). The answer of this category will be checked and graded by the e-examiner system, as shown in Figure 1-a.

\begin{tabular}{|c|c|c|}
\hline \multirow{5}{*}{$\begin{array}{l}\mathbf{1} \\
\mathrm{T}\end{array}$} & \multicolumn{2}{|r|}{$\begin{array}{l}\text { Which of the following languages have case-sensitive } \\
\text { names? }\end{array}$} \\
\hline & ב & $\mathrm{C}++$ \\
\hline & 口 & COBOL \\
\hline & Q & Fortran \\
\hline & ב & Pascal \\
\hline
\end{tabular}

b. Verifies whether or not the string can be generated by grammar. Answer: $\quad \square$ True $\square$ False

c. A......... determines the order in which operators of equal Precedence are evaluated when they occur in the same expression (Associativity, Availability, Precedence, Mandatory)

d. Consider the following XX grammar: LP $\rightarrow$ LP FUNC ( PAR ; PAR ) $\mid$ LP ( PAR ) $\mid$ exec FUNC $\rightarrow$ ID PAR $\rightarrow$ LP $\mid$ NUM

Note: (NUM is the token for numbers and ID is the token for identifiers).

Answer the following questions:

- Give the equivalent grammar after removing the left recursion from the above grammar

- $\quad$ Derive the following string from LP. exec $\operatorname{svg}(\operatorname{exec} \max (2 ; 5) ; 4)$

Fig. 1. Examples of Question Types stored in Question KB

- The True/False Category; where a question could be a text (sentence), while the answer should be one of two options (true or false), it is a logical choice. The answer of this category will be checked and graded by the eexaminer system. An example of this category is shown in Figure 1-b.

- The Fill in the Blanks Category; where a question could be a text (sentence), while the answer is oneword/simple-sentence to be selected from a pool-from 2 to 10- of words/small sentences. So, the process of filling in the spaces looks like matching certain question with a suitable answer. The answer of this category will be checked and graded by the e-examiner system. An example of this category is shown in Figure 1-c.

- The General Category; where a question could be in a form differs than each of the above 3 forms. It could be a long or short text, including graphs, equations, or numbers. Also, it could be imported from an external file. While the answer could be a free text including graphs, equation, or whatever the examined person want to write. Answer of this category of question should be checked and graded manually, by the examiner/ instructor. An example of this category is shown in Figure 1-d.

\section{MAIN COMPONENTS OF THE E-EXAMINER}

The proposed e-examiner system consists mainly, as shown in Figure 2, of four components; each component cooperates with each other to prove the system generality.

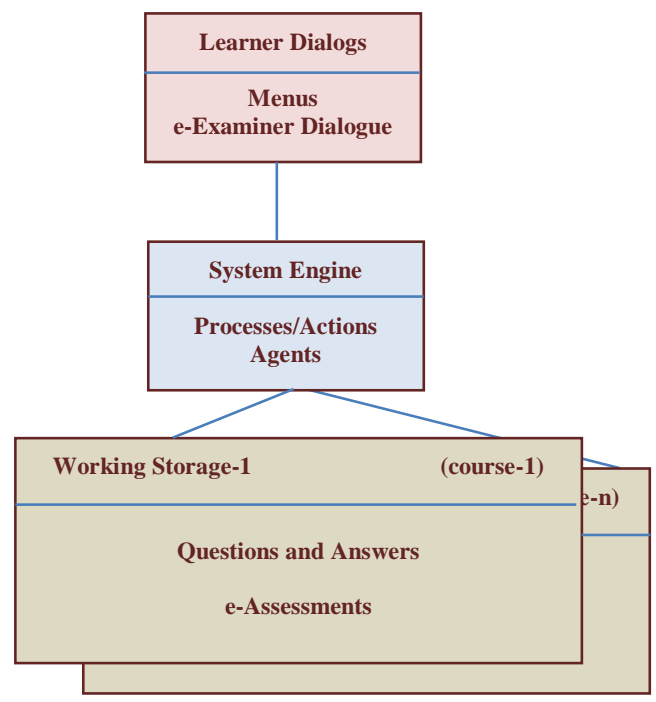

Fig. 2. Structure of the e-examiner system

- Learner Dialog; which is a menu-driven dialog for easing learner interaction with the e-examiner system directly. After finishing certain topics or certain course or part of course, he will navigate this dialog, thin the eexaminer will start creation of a personalized eassessment special to that learner. After passing answers of a learner to the e-examiner, it will check and grade his answers, and update the profile of that learner.

- System Engine; which involves four intelligent agents, as shown in Figure 3. The first agent is the control agent, which controls the process and agents. The second intelligent agent creates, fills and updates personal profiles of the examined learners. While the third agent will serve in selecting questions and create a personalized e-assessment and learns from the instructor examiner by watching him and gets from his experience, at editing questions or at creating exams. The fourth agent is the grading agent, which is responsible of checking and grading learner answers.

- Question Bank Knowledge Base; which is a semantic network KB, as shown in Figure 4. It consists of nodes like: exams, exercise, questions, answers and links (relations between nodes). Its structure is based on object oriented analysis of application domains. It holds an indexing structure used by the inference engine to locate questions. Each node or link is represented by an object of a suitable class.

- Working Storages; where each working storage is assigned to certain topic, chapter or course. The eexaminer system can be switched from one working storage to another. 


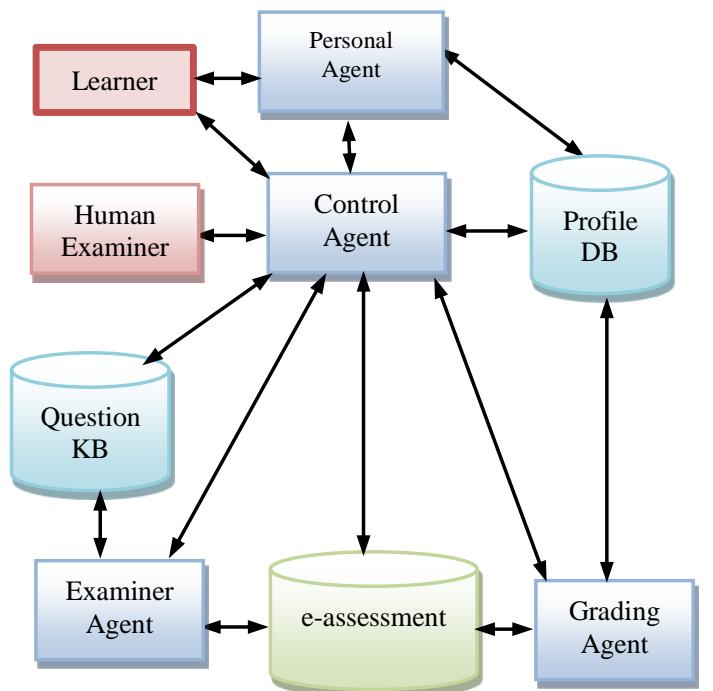

Fig. 3. Intelligent Agents in e-examiner system Agent

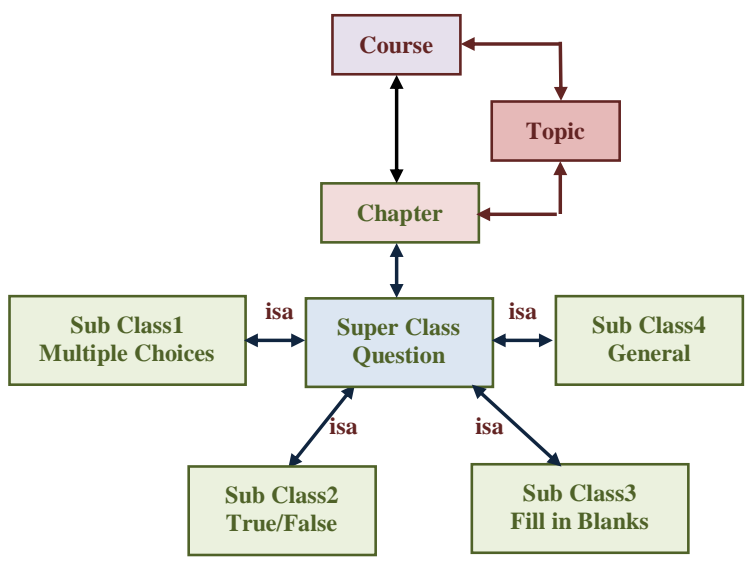

Fig. 4. Semantic Net of e-Examiner System

\section{BUILDING THE QUESTION KB}

A topic (course) material as application domain is analyzed using object oriented techniques to be used to create e-assessment. This e-assessment should cover that material completely or even a part(s) of it the learner profile and studying. Each domain is defined as objects like courses, topics, chapters, assessment exercises, questions, attributes, answers and relationships between these objects.

This question KB is designed, as shown in Figure 4, to be instantiated for several course, one course at a time. In the first use of the e-examiner, name of college or institute, department, and courses could be identified. Then, questions and their answered could be edited.

\section{A. Organization of the Question $K B$}

Questions to select from, should be distributed over the question $\mathrm{KB}$, which designed and organized in certain manner that enables the e-examiner to select questions randomly according the learner(s) profile and e-assessment factors, which are supervised by the intelligent agents.

At editing questions to be acquired, the editor will provide the KB with certain information to be used later as a basis for the e-examiner to select a set of questions for certain eassessment. It should takes in consideration, that the eassessment should cover majority or even specified parts of course material and should include various levels of difficulties.

The KB is designed to deal with the categories of question listed in the previous section. Its entity classes are fulfilled with attributes that lead it to be suitable to acquire the knowledge learned from the examiner at building eassessments. It has one Super Class and 4 Sub Classes, which has to be suitable to hold all categories of question listed in the previous section.

1) The Super Class question is the base for all categories of questions. It has some important attributes that are inherited by the other four sub classes. These attributes include a string attribute for the question text, pointer to the chapter or topic related to, and pointers to two lists of links (relations). It also, includes an attribute for learner answer of that question.

The first type of links are to the reminded questions questions that are suggested to be with that a certain question in the same e-assessment. While, the second type of links are to the rejected questions -questions that are not allowed to be with that a certain question in the same e-assessment. The other four sub classes should have the inherited attributes of the super class, in addition to special attributes as listed below.

2) The Sub Class for Multiple Choice Questions; which have many special attributes: four string attributes for predicted choices, and character attribute to denote the correct choice.

3) The Sub Class for True/False Questions; which have only one special attribute, which is a binary attribute. This attribute will hold the answer, which is 1 (for True question text) or 0 (for False Question text).

4) The Sub Class for Filling in Blanks Questions; which have only one special attribute, which is a string attribute to hold the correct text to be filled in the spaces.

5) The Sub-Class for General Questions; which have only one special attribute, to hold the complete answer of the generic question. Contents of this attributes could be imported from an external file. This answer could be edited or imported from a file.

\section{B. Filling the Question $K B$}

Question KB should be initiated for each course individually by certain information, like course code and name, department and/or institute offering that course; number of predicted chapter (could be modified latter by increasing or decreasing certain chapter). For each chapter, questions and their answers according to the four previous question types, listed before in section II, could be supplied to the system.

The human examiner starts supplying questions to the eexaminer. First, he selects the course, topic, chapter, and one question type of those listed in section II. Then, he supplies the question text, accompanied with all needed information as described in section III. Then, e-examiner will fill the KB 
tables with questions and their related knowledge.

Knowledge within the two lists of links could be acquired at entering a new question or be learnt from the human examiner when he add/rejects certain question.

\section{INTELLIGENT AGENTS AND AN E-ASSESSMENT}

The process of building an e-assessment should emphasize that each course include a number of chapters/topics. Some or all of these chapters could be covered by the e-assessment. This will help the e-examiner in selecting questions from the specified chapters or topics. Also, dividing a course into chapters helps to divide assessment into logical parts and "accumulate" assessment question KB (for example, you have 500 questions, but single e-assessment consists of 20 questions that are randomly selected from the $\mathrm{KB}$ ).

\section{A. Role of Agents in the e-Examiner System}

As seen in Figure 3, the e-examiner system has four agents; each of them has its important role in the main process of building and grading of an e-assessment, as described below:

- Control Agent (CA) is responsible of managing the process of building and grading of e-assessments. It controls and initiates other agents.

- Personal Agent (PA) is responsible of gathering information about learner and saving it in profile file. It also retrieves and updates his profile file.

- Examiner Agent (EA) is responsible of selection of question from question $\mathrm{KB}$. It uses random and mathematical functions in additions to the knowledge resides in the relations between different questions to pick the best choices from many enumerated possibilities. It manipulates questions and other objects and the in-between relations in a standard way with all presented topic(s) or chapter(s). It watches the examiner when he requests to remove/add certain questions from/to the e-assessment produced by the system (in the training phase). It has its actions on the knowledge in the relation between questions for next e-assessment building.

- Grading Agent (GA) is responsible of checking and grading e-assessments passed by certain learner(s). It compares answers of learner with answers stored with questions in question KB. Then, it updates profiles of examined learner(s), according to grading results.

\section{B. Criteria of an e-Assessment}

The presented e-examiner is a comprehensive solution for building e-assessments on the Internet and Intranet and grading those e-assessments. It can easily select all exercises types and format the text, add graphics, formulas in offline and online e-assessments.

In the offline e-assessments, the tool can produces an eassessment to be printed and copied to be distributed over students in a class. Also, it will produce a key for each eassessment, to help the examiner to check answers of the examined people, and grading their e-assessment.
E-examiner does the process of building an e-assessment, as shown in Fig. 5, it emphasized the following criteria:

- The heading interface of the e-assessment is fully customizable.

- E-examiner supports multi-language.

- Questions and answers could be shuffled randomly (for every particular question), to avoid any fraud by people who pass your tests.

- Unlimited number of questions in a single test and up to 4 answers in multiple choices.

- E-examiner system is trained in the beginning under supervision of the human examiner.

- Then the whole process is fully automated.

\section{Initiating the e-examiner System}

Before using the e-examiner system on-line with learners to generate e-assessment(s), it has to be initialized and trained to be ready for its work. Also, it will be adjusted to produce off line assessments.

For producing assessments using e-examiner system, the human examiner should initiate the assessment by supplying some information like the name, top introduction, bottom conclusion, and logo of the examining institute. Then, he should provide course name and chapters to be covered in the assessment to be selected from those available in the question KB.

Some optional data -could be applied to the system like: Academic year, Semester, Student sections, e-assessment duration, maximum score, e-assessment type (midterm, quiz, final), sort of e-assessment (A, B), total mark. While for online/offline assessment, the human examiner should specify the types of exercises in the assessment, number of question in each, and the mark specified for each exercise.

\section{BUILDING AND GRADING AN E-ASSESSMENT}

The e-examiner system can start production of offline/online assessments under the supervision the human examiner. Then it can generate e-assessment alone with no need to guidance from human examiner.

\section{A. Training of the e-Examiner by Human Examiner}

In first use of the e-examiner system, it should passes through a training session to build e-assessments under the supervision of a human examiner, as seen in the algorithm shown in Figure 5.

The EA agent will get its experience by watching the human examiners, in two different situations.

First, at editing a question; when the human examiner specifies that some question(s) are preferred to be in the same e-assessment or should prevented from being in the same eassessment with that question.

Secondly, at building an e-assessment, when the human examiner asks to remove certain question(s) or adds certain ones. 
1. PA agent GETS e-Assessment Data: Course, Chapters covered, From Learner(s) profile

2. CA agent GETS Exercise Data: number of exercises, type, number of questions in each exercise from Human examiner

3. CA agent CALCULATES Number of questions in each exercise from each Chapter.

4. For each exercise in the e-assessment Do

5. Do Until end of questions in the exercise EA agent SELECTS a question using Random function \& Links

EA agent CHECKS Reminding \& Rejection links in the relationships of the question KB net.

6. CA agent DISPLAY Complete exercise to the human examiner.

7. IF NOT APPROVED (by human examiner)

8. EA agent GETS rejections and reasons, suggestions and reasons (reminding), UPDATE links of the question $\mathrm{KB}$, and UPDATES exercise.

9. ELSE CONTINUE (go to 4)

10. CA agent CONSULTS complete e-Assessment to human examiner

Fig. 5. Algorithm for Training the e-Examiner System

At beginning a session to build an e-assessment from the question $\mathrm{KB}$, e-examiner system performs its task through several steps, which incorporates knowledge acquisition and learning with knowledge retrieval from its question $\mathrm{KB}$, based on intelligent agents.

The e-examiner asks the examiner about type of each exercise and its number of questions. It calculates number of questions per each chapter by mathematical functions.

Then, EA agent starts a process of selection questions, one by one, until finishing selecting. This selection is based on random function and according to the reminding and rejecting knowledge resides in the links between each question and other questions in the question KB.

The e-examiner consults selection results in a complete exercise to the human examiner for approval. Sometimes, she/he removes certain question or adds another question. In this case, the EA agent asks her/him for explanation.

This explanation is acquired to a rejecting knowledge in case of refusing certain question, and to a reminding knowledge in case of adding certain questions. This knowledge will reside in the links between any requested question and other questions in the question KB.

After performing the requested modification, the tool, finally, produces a file including the final form of the eassessment or the quiz. This e-assessment version should be reviewed by the human examiner. Also, the e-examiner system produces a key for questions.

\section{B. The e-Examiner Builds an e-Assessment}

After the e-examiner passed about 50 training sessions in building e-assessments, under the supervision of a human examiner, it is ready to build its own e-assessment, depending on his knowledge. It builds its e-assessments, as shown in Figure 6, depending on the learner profile data and the regulations acquired from before the human examiner.

\section{C. e-Examiner Grades an e-Assessment}

The grading process of an e-assessment is done so easily by the e-examiner system. At selecting certain question to add to an e-assessment, the EA agent retrieves its answer from the question $\mathrm{KB}$, attached with it. Then the GA agent compares the answer of learner with the answer retrieved from the question KB. If they are the same, it accumulates a one degree to the total degrees collected by the learner in that eassessment. Finally, it gives the total degree to the PA agent to update the learner profile data.

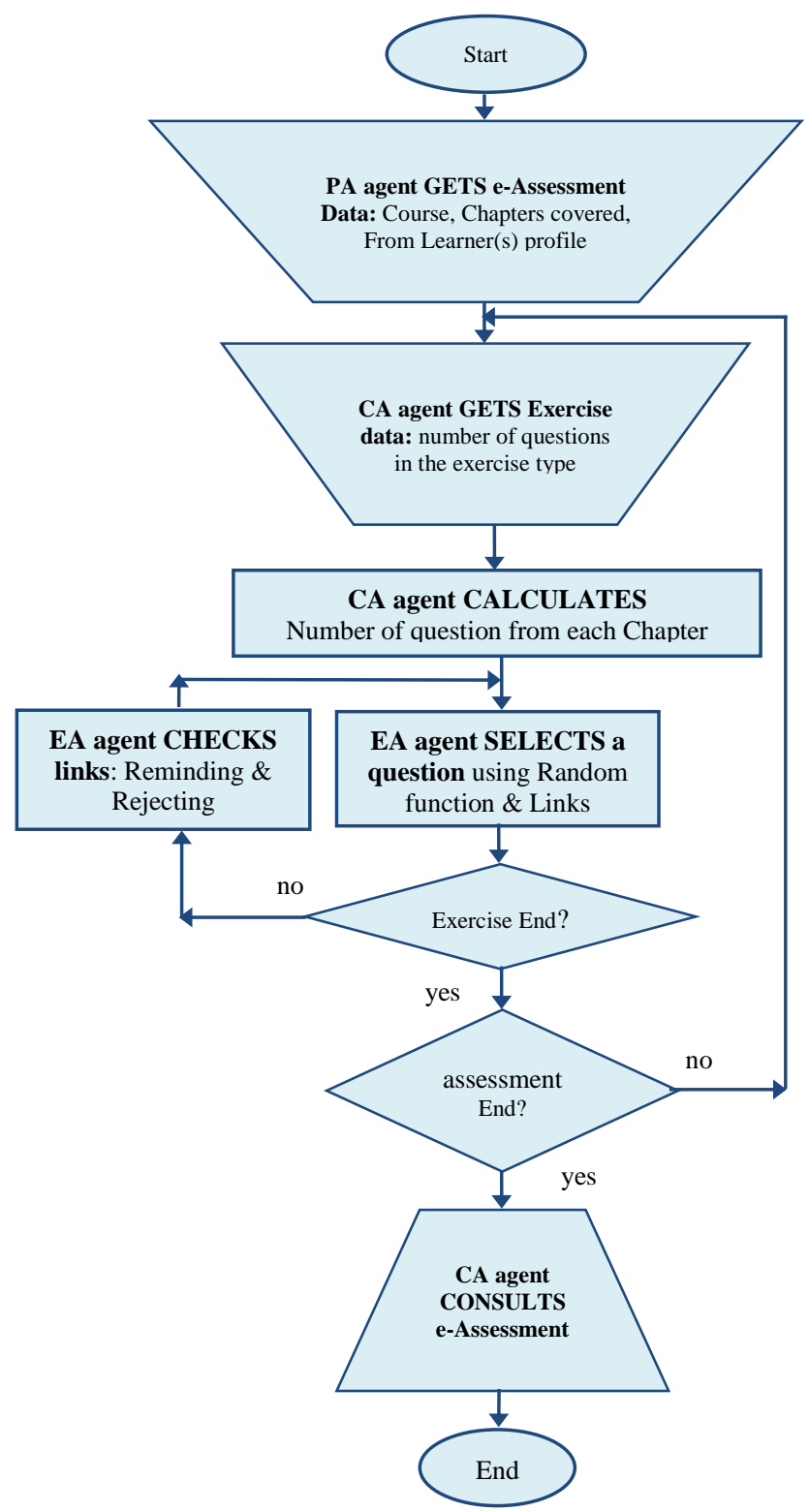

Fig. 6. Building an e-assessment by the e-examiner system

\section{CONCLUSIONS}

The presented paper presented a design of a smart eexaminer system, which is running four intelligent agents on a semantic net of question KB. Its agents; control agent, personal agent, examiner agent, and grading agent co-operated 
together to perform its task of producing and grading personalized e-assessment, depending on learner studying and level.

The system should start its work under the supervision of human examiner. The examiner agent (EA) got experience by watching the human examiner, in two different situations: at editing a question; when the he specifies that some question(s) are preferred to/not-to be prevented from being in the same eassessment with that question, and at building e-assessment; when the examiner asks to remove certain question(s) or add certain ones.

The e-examiner system could be used on various education level and nature to generate e-assessments for employees and students in training courses. Also, it produced a key for the produced e-assessment, to be used in grading, and giving final marks of e-assessments. Future work will include enhancing system architecture and improving its security and reading IP address of learner.

\section{REFERENCES}

[1] Zhi Liu \& Bo Chen, "Model and Implement an Agent Oriented ELearning System", the International Conference on Computational Intelligence for Modeling, Control and Automation, and International Conference Intelligent Agents, Web Technologies and Internet Commerce, Vienna (CIMCA-IAWTIC'05) 0-7695-2504-0/05, 28-30 Nov.2005, pp. 859-864. http://doi.ieeecomputersociety.org/10.1109/CIMCA.2005.1631576

[2] J. Anderson, F. Boyle, A. Corbett, and M. Lewis, "Cognitive Modeling and Intelligent Tutoring", Elsevier Science Publishers, Journal Artificial Intelligence, Special issue on artificial intelligence and learning environments archive, vol. 42, no. 1, pp. 7-49, Feb. 1990. http://act- r.psy.cmu.edu/wordpress/wpcontent/uploads/2012/12/119CogMod_IntTut.pdf

[3] Jin-Ling Lin and Ming-Hung Chen, "An Intelligent Agent for Personalized E-Learning", $8^{\text {th }}$ Inter. Conference on Intelligent Systems Design and Applications - Volume 01, Kaohsiung, 26-28 Nov. 2008, pp. 27-31. http://ieeexplore.ieee.org/xpl/articleDetails.jsp?arnumber $=4696172$

[4] S. J. Russell, and P. Norvig, (2009, Dec. 11), Artificial Intelligence - A Modern Approach, $3^{\text {rd }}$ Ed., Prentice Hall. http://stpk.cs.rtu.lv/sites/all/files/stpk/materiali/MI/Artificial\%20Intellig ence\%20A\%20Modern\%20Approach.pdf

[5] M. Niazi and A. Hussain, "Agent-based Computing from Multi-agent Systems to Agent-Based Models: A Visual Survey". Springer Scientometrics, vol. 89, no. 2, pp. 479-499, 2011. doi: 10.1007/s11192011-0468-9.

[6] S. A. Agarwal and M. Sinha," Multi Agent Adaptive E-Learning System", International Journal of Advanced Research in Computer Science and Software Engineering, vol. 3, no. 7, pp. 242-246, IJARCSSE, July 2013.

[7] Advanced eLearning Builder, "Authoring tool designed for creating elearning materials", 2015. http://www.eduiq.com/elearning.htm

[8] MicroGrade and MicroTest,"desktop applications", 2015. http://www.chariot.com/

[9] OnlineTesting,"software and programming services", 2015. http://www.onlinetesting.net/

[10] ProfProf, "Create Online Tests \& Quizzes Easily", 2015. http://www.proprofs.com/quiz-school/

[11] AditSofware," Exams, Tests and Quizzes Made Easy", 2015. http://www.aditsoftware.com/

[12] Quizworks, " Build online exams with our fun \& easy to use tool", 2015. "https://www.onlineexambuilder.com/

[13] ExamJet Quiz Maker," Quiz maker software made with love", 2015. https://www.igneon.com/ 